\title{
Detection of long repeat expansions from PCR-free whole-genome sequence data
}

Egor Dolzhenko, ${ }^{1,18}$ Joke J.F.A. van Vugt, ${ }^{2,18}$ Richard J. Shaw, ${ }^{3,4}$ Mitchell A. Bekritsky, ${ }^{3}$ Marka van Blitterswijk, ${ }^{5}$ Giuseppe Narzisi, ${ }^{6}$ Subramanian S. Ajay, ${ }^{1}$ Vani Rajan, ${ }^{1}$ Bryan R. Lajoie, ${ }^{1}$ Nathan H. Johnson, ${ }^{1}$ Zoya Kingsbury, ${ }^{3}$ Sean J. Humphray, ${ }^{3}$ Raymond D. Schellevis, ${ }^{2}$ William J. Brands, ${ }^{2}$ Matt Baker, ${ }^{5}$ Rosa Rademakers, ${ }^{5}$ Maarten Kooyman, ${ }^{7}$ Gijs H.P. Tazelaar, ${ }^{2}$ Michael A. van Es, ${ }^{2}$ Russell McLaughlin, ${ }^{8,9}$ William Sproviero, ${ }^{10}$ Aleksey Shatunov, ${ }^{10}$ Ashley Jones, ${ }^{10}$ Ahmad Al Khleifat, ${ }^{10}$ Alan Pittman, ${ }^{11}$ Sarah Morgan, ${ }^{11}$ Orla Hardiman, ${ }^{8,9}$ Ammar Al-Chalabi, ${ }^{10}$ Chris Shaw, ${ }^{10}$ Bradley Smith, ${ }^{10}$ Edmund J. Neo, ${ }^{10}$ Karen Morrison, ${ }^{12}$ Pamela J. Shaw, ${ }^{13}$ Catherine Reeves, ${ }^{6}$ Lara Winterkorn, ${ }^{6}$ Nancy S. Wexler, ${ }^{14,15}$ The US-Venezuela Collaborative Research Group, ${ }^{16}$ David E. Housman, ${ }^{17}$ Christopher W. Ng, ${ }^{17}$ Alina L. Li, ${ }^{17}$ Ryan J. Taft, ${ }^{1}$ Leonard H. van den Berg, ${ }^{2}$ David R. Bentley, ${ }^{3}$ Jan H. Veldink, ${ }^{2,18}$ and Michael A. Eberle ${ }^{1,18}$

${ }^{1}$ Illumina Incorporated, San Diego, California 92122, USA; ${ }^{2}$ Department of Neurology, Brain Center Rudolf Magnus, University Medical Center Utrecht, Utrecht University, 3584 CX Utrecht, The Netherlands; ${ }^{3}$ Illumina Limited, Chesterford Research Park, Little Chesterford, Nr Saffron Walden, Essex, CB10 1XL, United Kingdom; ${ }^{4}$ Repositive Limited, Future Business Centre, Cambridge CB4 2HY, United Kingdom; ${ }^{5}$ Department of Neuroscience, Mayo Clinic, Jacksonville, Florida 32224, USA; ${ }^{6}$ New York Genome Center, New York, New York 10013, USA; ${ }^{7}$ SURFsara, 1098 XG Amsterdam, The Netherlands; ${ }^{8}$ Academic Unit of Neurology, Trinity College Dublin, Trinity Biomedical Sciences Institute, Dublin 2, Republic of Ireland; ${ }^{2}$ Department of Neurology, Beaumont Hospital, Dublin 9 , Republic of Ireland; ${ }^{10}$ Department of Basic and Clinical Neuroscience, Maurice Wohl Clinical Neuroscience Institute, King's College London, London SE5 9RX, United Kingdom; ${ }^{11}$ Department of Molecular Neuroscience, UCL Institute of Neurology, London WC1N 3BG, United Kingdom; ${ }^{12}$ University of Southampton, Southampton SO1 7 1BJ, United Kingdom; ${ }^{13}$ Sheffield Institute for Translational Neuroscience, University of Sheffield, Sheffield S10 2HQ, United Kingdom; ${ }^{14}$ Columbia University, New York, New York 10032, USA;

${ }^{15}$ Hereditary Disease Foundation, New York, New York 10032, USA; ${ }^{16}$ The US-Venezuela Collaborative Research Group;

${ }^{17}$ Department of Biology, Massachusetts Institute of Technology, Cambridge, Massachusetts 02139, USA

Identifying large expansions of short tandem repeats (STRs), such as those that cause amyotrophic lateral sclerosis (ALS) and fragile $\mathrm{X}$ syndrome, is challenging for short-read whole-genome sequencing (WGS) data. A solution to this problem is an important step toward integrating WGS into precision medicine. We developed a software tool called ExpansionHunter that, using PCR-free WGS short-read data, can genotype repeats at the locus of interest, even if the expanded repeat is larger than the read length. We applied our algorithm to WGS data from 3001 ALS patients who have been tested for the presence of the C9orf72 repeat expansion with repeat-primed PCR (RP-PCR). Compared against this truth data, ExpansionHunter correctly classified all $(212 / 212,95 \% \mathrm{Cl}[0.98,1.00])$ of the expanded samples as either expansions (208) or potential expansions (4). Additionally, 99.9\% (2786/2789, 95\% Cl [0.997, 1.00]) of the wild-type samples were correctly classified as wild type by this method with the remaining three samples identified as possible expansions. We further applied our algorithm to a set of 152 samples in which every sample had one of eight different pathogenic repeat expansions, including those associated with fragile X syndrome, Friedreich's ataxia, and Huntington's disease, and correctly flagged all but one of the known repeat expansions. Thus, ExpansionHunter can be used to accurately detect known pathogenic repeat expansions and provides researchers with a tool that can be used to identify new pathogenic repeat expansions.

[Supplemental material is available for this article.]

\footnotetext{
${ }^{18}$ These authors contributed equally to this work.

Corresponding authors: meberle@illumina.com,

J.H.Veldink@umcutrecht.nl

Article published online before print. Article, supplemental material, and publication date are at http://www.genome.org/cgi/doi/10.1101/gr.225672.117.

Freely available online through the Genome Research Open Access option.
}

○ 2017 Dolzhenko et al. This article, published in Genome Research, is available under a Creative Commons License (Attribution 4.0 International), as described at http://creativecommons.org/licenses/by/4.0/. 
Variant callers for small variants such as single-nucleotide polymorphisms and small insertions or deletions typically require multiple reads to completely span the full length of the nonreference allele (DePristo et al. 2011; Raczy et al. 2013). For variants that deviate significantly from the reference, alternative methods such as de novo assembly can be used if the variant is not highly repetitive (Iqbal et al. 2012; Weisenfeld et al. 2014; Li 2015; Chen et al. 2016). Because high-throughput WGS technologies are currently limited to $\sim 150$ base pair (bp) read lengths, variantcalling methods that rely on reads aligned to the reference are subsequently limited to repeat lengths less than 150 bases (Narzisi and Schatz 2015). Many pathogenic repeat expansions have repeats spanning hundreds to thousands of base pairs (Dürr et al. 1996; Gatchel and Zoghbi 2005; Kronquist et al. 2008; Gijselinck et al. 2016), so it has been assumed that short-read sequencing technologies may not be able to identify pathogenic repeat expansions (Loomis et al. 2013; Ashley 2016).

A recently discovered hexamer (GGCCCC) repeat expansion in the C9orf72 locus is a major cause of both ALS and frontotemporal dementia (DeJesus-Hernandez et al. 2011; Renton et al. 2011; Gijselinck et al. 2012). In particular, the pathogenic repeat length (more than 30 repeats; $>180 \mathrm{bp}$ ) is present in $\sim 10 \%$ of all ALS patients including $\sim 40 \%$ of familial ALS cases and $\sim 6 \%-8 \%$ of sporadic ALS cases in some populations (DeJesus-Hernandez et al. 2011; Renton et al. 2011; Gijselinck et al. 2012). The most widely used method to detect C9orf72 repeat expansions is repeat-primed PCR (RP-PCR) together with fragment length analysis (Akimoto et al. 2014). Interpretation of these PCR results can be challenging due to indels in the flanking regions of the repeat, which can lead to both false positives and false negatives (Akimoto et al. 2014). In addition, these PCR techniques do not provide an estimate of the length of the repeat expansions. Southern blotting is the current gold standard for estimating repeat length, but this method is very challenging to set up; requiring a significant amount of input DNA (generally $10 \mu \mathrm{g}$ ) and suffering from imprecise size estimates due to somatic heterogeneity (Buchman et al. 2013; Akimoto et al. 2014). As WGS is widely adopted for use in precision medicine initiatives (Ashley 2015, 2016; Marx 2015) and large-scale research projects, a reliable method is needed that can identify the presence or absence of potentially pathogenic repeat expansions in WGS data and also determine their approximate length without additional tests.

Here, we present a method to genotype STRs from PCRfree, WGS data implemented in a software package named ExpansionHunter. This method can determine the approximate size of repeats from just a few units in length up to large, pathogenic expansions that may be significantly longer than the read length. To quantify the performance of this algorithm, we first estimate the repeat lengths of two cohorts of ALS patients, all of whom were independently assessed for the presence of the pathogenic C9orf72 repeat expansion using RP-PCR, and determine the overall sensitivity and specificity of the assay. In addition, we also demonstrate that this method is generally applicable for detecting other repeat expansions by applying it to a set of 152 samples harboring eight other repeat expansions including those that cause fragile X syndrome, Friedreich's ataxia, and Huntington's disease. We also demonstrate the improved accuracy of this method for genotyping STRs shorter than the read length compared to an existing method (lobSTR) on 860 samples for which the size of the longest repeat allele had been experimentally determined. These analyses show that ExpansionHunter is a comprehensive tool for genotyping both short and long repeats. Thus, it can be used to test for the presence of known pathogenic repeat expansions and can be extended as a general STR caller to identify novel pathogenic expansions in population and pedigree studies.

\section{Results}

We performed paired-end, PCR-free, WGS at an average depth of 45x using Illumina HiSeq 2000 (100 bp reads) and Illumina HiSeq X (150 bp reads) systems on two cohorts of patients with ALS (Methods). The first cohort of 2559 patients was used during the development of ExpansionHunter to test the implementation of the algorithm for bugs and to calculate off-target regions (although the core algorithm and its parameters were not informed by these data). The second cohort of 442 patients was used to validate the implementation of the program. All 3001 samples were tested for presence of the C9orf72 repeat expansion with RP-PCR (Methods). A second RP-PCR test using a different primer set, fragment length analysis, and Southern blotting was performed on 71 samples from the initial cohort, of which 55 had a pathogenic C9orf72 repeat according to the first RP-PCR (Supplemental Table 2). Additionally, the fluorescent PCR plots were reevaluated for a subset of the samples (Supplemental Fig. 6). As explained in the section on pathogenic C9orf72 repeat expansion determination, some of the original RP-PCR calls were deemed incorrect based on this reassessment and changed accordingly (Supplemental Table 2), bringing the total counts of expanded and wild-type samples to 212 and 2789 , respectively.

To quantify repeat lengths, we developed an algorithm that identifies reads that either (1) fully span the repeat (spanning reads); or (2) include the repeat and the flanking sequence on one side of the repeat (flanking reads); or (3) are fully contained in the repeat ("in-repeat" reads or IRRs) (Fig. 1). For repeats shorter than the read length of the sequence data we calculate the repeat length using spanning and flanking reads (Fig. 1). To estimate the lengths of repeats that are longer than the read length, we identify and count the IRRs. There are three main hurdles associated with using IRRs to accurately identify repeat expansions that exceed read lengths: (1) identifying IRRs comprised of a potentially errorprone repeat motif; (2) identifying regions in the genome where paired IRRs are systematically (and possibly incorrectly) placed by the aligner; and (3) estimating the repeat length based on the total number of IRRs identified. Here, we describe how we solve these problems to identify and characterize expanded repeats accurately.

\section{On-target IRRs}

Identifying reads originating in highly repetitive regions can be difficult because sequencing error rates are higher in low complexity regions such as homopolymers and STRs (Benjamini and Speed 2012), so we implemented a weighted measure that penalizes base mismatches at low-quality bases less than mismatches at highquality bases (Methods). To identify IRRs that originate within the C9orf72 repeat, we extracted all read pairs in which one read is an IRR and the other read aligns with high accuracy (mapping quality [MAPQ] at least 60 ) within $1 \mathrm{kbp}$ of the C9orf72 repeat locus. We call such reads anchored IRRs. Because the mates of anchored IRRs align uniquely near the target repeat, we are confident that the IRRs come from the C9orf72 repeat locus. Anchored IRRs can be used to estimate the size of repeats that are longer than the read length but shorter than the fragment length. For repeats exceeding the fragment length, the number of anchored IRRs provides a lower bound for the repeat length. 


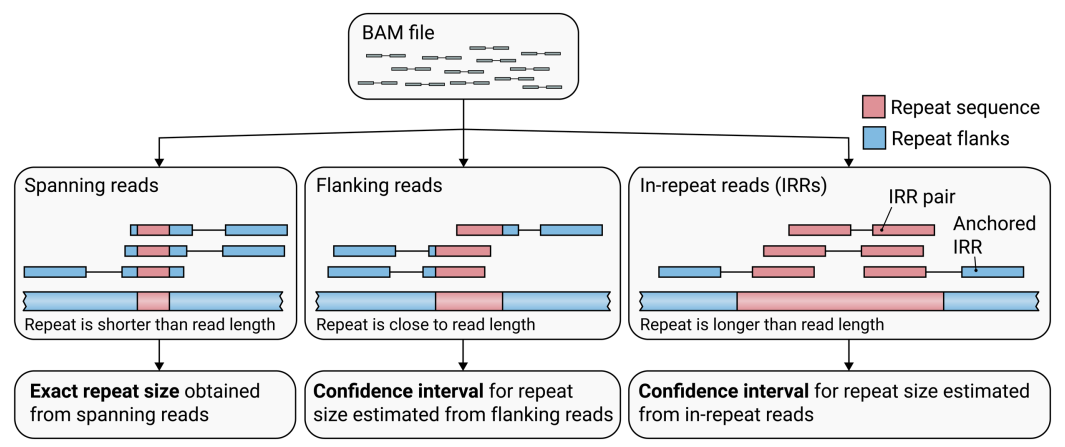

Figure 1. An outline of how ExpansionHunter catalogs reads associated with the repeat locus of interest and estimates repeat lengths starting from a binary alignment/map (BAM) file. (Left) Exact sizes of short repeats are identified from spanning reads that completely contain the repeat sequence. (Middle) When the repeat length is close to the read length, the size of the repeat is approximated from the flanking reads that partially overlap the repeat and one of the repeat flanks. (Right) If the repeat is longer than the read length, its size is estimated from reads completely contained inside the repeat (inrepeat reads). In-repeat reads anchored by their mate to the repeat region are used to estimate the size of the repeat up to the fragment length. When there is no evidence of long repeats with the same repeat motif elsewhere in the genome, pairs of in-repeat reads can also be used to estimate the size of long (greater-than-fragment-length) repeats.

\section{Off-target IRRs}

The library preparation used for these sequencing experiments had a mean fragment size of $\sim 350-450 \mathrm{bp}$, but the C9orf72 repeat expansion can be $>10 \mathrm{kbp}$ in length (Gijselinck et al. 2016). This means that in addition to anchored IRRs, pairs in which both mates are IRRs could be present in samples with the C9orf72 repeat expansion (Fig. 1). Because the expanded repeat is not present in the reference, these paired IRRs may not align to the C9orf72 repeat locus and could either not align at all or misalign to a different locus in the genome (Church et al. 2015; Gijselinck et al. 2016). To identify unaligned or misaligned IRRs, we tested every $(\mathrm{MAPQ}=0)$ read in all 182 expanded ALS samples of the first cohort identified by the first round of RP-PCR as having the C9orf72 repeat expansion. These 182 samples contained 29,619 poorly mapped paired IRRs altogether $-33 \%$ of these were unaligned and $67 \%$ resided in 29 loci (which we term off-target regions), and only $0.1 \%$ were located elsewhere (Methods). Conversely, when we performed the same analysis on 182 random samples without the C9orf72 repeat expansion according to RP-PCR, we did not find paired IRRs in any genomic locus.

We next analyzed positions where the mates of anchored IRRs aligned in all 2559 samples from cohort one. For each sample, we identified all the anchored IRRs and then grouped the IRRs anchored within $500 \mathrm{bp}$ of one another. The C9orf72 repeat locus had many anchored IRRs in nearly all samples with a pathogenic repeat expansion (178 samples had five or more anchored IRRs and 160 had 10 or more) indicating that the repeat exceeds the read length in these samples as expected. Only 10 genomic loci had more than one IRR anchored outside of the C9orf72 repeat locus in any of these samples (Fig. 2). Based on this, we considered all paired IRRs to originate from the C9orf72 repeat locus and included them in the size estimation when testing this repeat.

\section{Repeat size estimation}

Improvements to short-read sequencing technology such as PCR-free sample preparation minimize the GC bias that previously bedeviled PCR-based WGS data (Meienberg et al. 2016). This is illustrated by the improved coverage of high GC regions such as the FMR1 repeat (Supplemental Fig. 5). These improvements enabled us to estimate the length of a region by the number of reads that originate from it even for regions with high GC content. By assuming that the number of reads that originate in a given region follows a binomial distribution, we were able to estimate the size of the repeat by the number of IRRs. The number of IRRs in individual samples ranged from 0 to 1314 corresponding to estimated C9orf72 repeat sizes of up to $7152 \mathrm{bp}$.

For shorter alleles, the sizes of repeats were determined using spanning reads (Fig. 1). For repeats that are close to the read length, the repeat may be too long to produce spanning reads but too short to produce IRRs. Therefore, the algorithm also uses flanking reads (Fig. 1) to estimate the repeat size (Methods). In the 2559 samples of cohort one, $1.6 \%$ (40) of the samples had a repeat size estimated using only flanking reads that resulted in repeat size estimates from 18 to 144 bp (Supplemental Table 2).

ExpansionHunter computes the maximum-likelihood genotype consisting of candidate repeat alleles determined by spanning, flanking, and in-repeat reads (Methods). When both alleles are longer than the read length, the algorithm computes intervals for possible sizes of short and long repeats based on the two extreme cases: (1) All reads come from one haplotype; or (2) half the reads come from each haplotype.

\section{Pathogenic C9orf72 repeat expansion detection}

The C9orf72 repeat sizes for both ALS cohorts determined by our method were compared to the RP-PCR results (Supplemental Table 2). Cases in which the estimated confidence interval for repeat size overlapped the pathogenic C9orf72 repeat size cutoff (i.e., the lower bound was less than 30 repeats and the upper bound was greater than 30 repeats) were defined as "gray" and

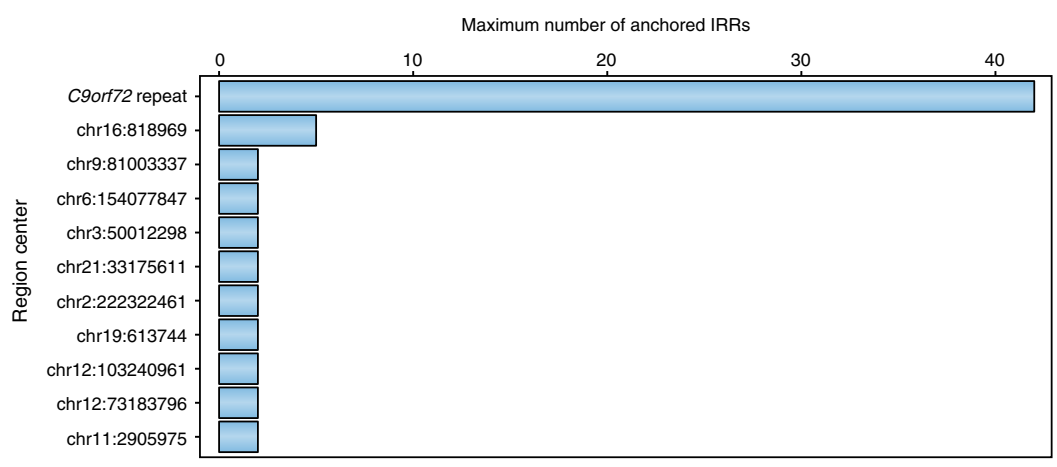

Figure 2. The maximum number of anchored IRRs observed in any of the 2559 samples from cohort one for the genomic loci with at least two anchored IRRs in at least one sample (Methods). 
considered "long" in all sensitivity/specificity calculations. Using the original RP-PCR calls as the ground truth, we identified 11 discrepant calls between our method and RP-PCR, resulting in the overall sensitivity and specificity of $98.6 \%$ and $99.6 \%$, respectively, for the WGS-based calls. Because RP-PCR involves many manual steps and, as has been shown previously, could be prone to error (Akimoto et al. 2014), we performed an additional analysis of the discrepant calls to understand the source of these conflicts.

Of the 11 samples with a discrepant classification between our method and the RP-PCR calls, eight were "EH positive/RPPCR negative" (positive $=$ expansion; negative $=$ normal); however, each of these discrepant calls had at least 13 anchored IRRs, which constitutes strong supporting evidence for a pathogenic repeat expansion in these samples (Supplemental Table 4; Supplemental Fig. 6). Predicting the repeat length using only the anchored reads also supported the pathogenic repeat expansion sizing in all eight "EH positive/RP-PCR negative" samples. Conversely, two of the three "EH negative/RP-PCR positive" samples had compelling read-level evidence supporting their negative status: The read-level data supported repeat alleles of two distinct sizes, each spanning fewer than 30 repeat units. Specifically, one sample contained 10 spanning reads with a repeat of size 2 and 10 spanning reads with a repeat of size 5 , whereas the other sample had a size estimate just under the pathogenic cutoff (16 to 26 repeat units). The final "EH negative/RP-PCR positive" sample had just one allele identified (consisting of two repeat units), but the number of spanning reads (38) was consistent with the read depth (mean depth $=44 \times$ ) in this sample supporting a homozygous, nonpathogenic variant (Supplemental Table 4; Supplemental Fig. 6).

We also reevaluated the original RP-PCR calls for eight samples (Supplemental Fig. 6) and performed an additional RP-PCR and fragment length analysis for 71 samples (Supplemental Table 2). This analysis showed that in 10 of the 11 conflicting calls, the original RP-PCR call was incorrect; therefore, ExpansionHunter and the RP-PCR results were consistent (Supplemental Table 4). The remaining conflict was also resolved after an additional RP-PCR was performed on this sample with different primers (Methods). Additionally, one sample classified as gray range by ExpansionHunter was reclassified from RP-PCR positive to RPPCR negative. Fragment length analysis estimated the repeat in this sample to be between 28 and 30 repeat units. Because we count gray range samples as expanded, we now consider this sample as misclassified by our analysis, although the experimental size range overlaps the size range predicted by ExpansionHunter. The remaining 67 samples had no conflicts between ExpansionHunter and RPPCR in either the first or second RP-PCR analysis. Reclassifying the original calls based on this additional analysis, the total number RP-PCR expanded samples increased from 208 to 212.

Comparing our calls against the updated RP-PCR results showed that the only discrepancies in classification are due to the seven "gray" calls, in which the samples likely have repeat lengths close to 30 repeat units (Table 1 ). Because we consider "gray" calls as expanded, this method produced just three false positives (EH gray/RP-PCR negative) and no false negatives. Overall, ExpansionHunter correctly flagged all (212/212, 95\% CI $[98.7 \%, 100 \%])$ of the expanded samples as either expansions (208) or potential expansions (4). Additionally, 99.9\% (2,786/ $2,789,95 \%$ CI $[99.7 \%, 100 \%])$ of the nonexpanded samples were correctly classified, and the three discrepant calls were labeled as "gray" by ExpansionHunter.

\section{Repeats shorter than the read length}

To quantify the accuracy of our method for alleles shorter than the read length, we compared our results to those obtained on 860 samples for which the size of the longest allele was estimated using fragment length analysis (Supplemental Table 2). In addition, we also analyzed these samples using the STR calling tool lobSTR (Gymrek et al. 2012). It should be noted that lobSTR is designed for general genome-wide STR calling based on spanning reads and is limited to calling repeat lengths shorter than the read length, so it may not make a call for longer repeats. In this comparison, the ExpansionHunter calls agreed with the fragment length analysis in 821 (95.5\%) of the samples, and the lobSTR calls agreed with the fragment length analysis in $734(85.3 \%)$ of the samples. Of the 39 ExpansionHunter repeat sizes that did not agree with the fragment length analysis, $20(51 \%)$ were in agreement with the lobSTR calls, and the remaining 19 calls were predicted to be longer repeats (spanning eight or more repeat units) where lobSTR is less likely to make a call (Supplemental Tables 2, 5).

Next, we analyzed the 1770 samples that were sequenced with $2 \times 150$ bp reads to get the distribution of the repeat lengths identified from spanning reads in the C9orf72 repeat. The distribution determined by this analysis is very similar to the results obtained in a previous study (van der Zee et al. 2013) that used an alternative repeat-primed PCR assay and a short tandem repeat (STR) fragment length assay with flanking primers optimized for alleles with high GC content (STR-PCR) allowing exact sizing of

Table 1. Sensitivity and specificity of C9orf72 repeat expansion detection by ExpansionHunter (EH) on the ALS samples taking the updated RPPCR results as the ground truth

\begin{tabular}{lcccc}
\hline & \multicolumn{2}{c}{ First cohort } & & Second cohort \\
\cline { 2 - 4 } & RP-PCR positive & RP-PCR negative & & RP-PCR positive \\
\hline EH positive & 181 & 0 & 27 & RP-PCR negative \\
EH negative & 0 & 2373 & 0 & 0 \\
EH gray & 3 & 2 & 1 & 1 \\
Sensitivity & $100 \%$ & - & $95 \% \mathrm{Cl}[87.7,100]$ & - \\
Specificity & $95 \% \mathrm{Cl}[98.0,100]$ & $99.9 \%$ & - & $99.8 \%$ \\
& - & $95 \% \mathrm{Cl}[99.7,100]$ & & $95 \% \mathrm{Cl}[98.7,100]$ \\
\hline
\end{tabular}

EH/RP-PCR positive (negative) category refers to samples classified as having an expanded (nonexpanded) C9orf72 repeat by each method. EH gray calls have confidence intervals overlapping the pathogenic cutoff (30). Gray calls were considered expanded when calculating sensitivity and specificity. 
normal lengths (Fig. 3). This indicates that we can accurately resolve the length of the short repeats. Because of the requirement for reads to span the STR fully, the maximum repeat size called by lobSTR is 11 repeats, although $4.2 \%$ (145 of 3394) of our alleles are sized greater than 11 repeats.

\section{Applying ExpansionHunter to other repeat expansions}

In addition to the C9orf72 repeat, several other pathogenic repeat expansions have been identified (McMurray 2010). To demonstrate the general applicability of our method, we tested eight other pathogenic repeat loci by sequencing and genotyping 152 samples with known expansions and 26 controls. The sample set contains 98 Coriell samples (https://catalog.coriell.org) from 64 families with a variety of repeat expansions associated with dentatorubral-pallidoluysian atrophy (DRPLA, ATN1 gene), fragile X Syndrome (FXS, FMR1 gene), Friedreich's ataxia (FRDA, FXN gene), Huntington's disease (HD, HTT gene), myotonic dystrophy type 1 (DM1, DMPK gene), spinocerebellar ataxia type 1 (SCA1, ATXN1 gene), spinocerebellar ataxia type 3 (SCA3, ATXN3 gene), and spinal and bulbar muscular atrophy (SBMA, $A R$ gene). In addition to the Coriell samples, our data include 54 samples with HTT expansions obtained from a rural fishing village in Venezuela with the highest concentration of Huntington's disease in the world (Wexler et al. 2004). These 54 samples were processed with a different alignment software (Li and Durbin 2009) allowing us to demonstrate that ExpansionHunter is compatible with other commonly used short-read aligners.

Taken together, these 152 samples represent different repeats with a variety of repeat sizes including normal, premutated, and fully expanded repeats. Premutated repeats are nonpathogenic repeats that are predisposed to become pathogenic/fully expanded in subsequent generations. Normal/premutation transitions for the repeats that we target ranged between 87 and 165 bases and premutation/full expansion transitions ranged between 114 and 600 bases. The repeats in the HTT, ATXN1, and AR genes are short enough that anchored IRRs alone are sufficient to detect the expansion. For the expansion in the FMR1 gene, we included off-target reads using the methodology we developed for the C9orf72 repeat to improve our ability to quantify large repeats. We did not include off-target locations for the other, potentially long repeats because the corresponding motifs (CAG and AAG) are common enough that we could not resolve which repeat the paired IRRs originated from.
Figure 4 depicts the sizes of the longer repeat allele determined by ExpansionHunter. Each of the 152 samples was tested for eight repeat expansions, one of which is expected to be expanded and the rest wild type. All 24 control samples were similarly tested across all eight expansions. Our method identified all repeats expected to be premutated (orange circles) or fully expanded (red circles). The categorization was correct for all repeats with an exception of the FMR1 repeats, in which 15 of 16 repeats were estimated by ExpansionHunter to be premutations instead of full expansions, and one ATXN1 expanded sample was identified in the normal range.

Although we correctly identified all but one of the expansions, there was one "control" sample showing the FXN expansion and three "control" samples with the FMR1 repeat size at the low end of the premutation range. Both of these results are unsurprising due to the higher carrier frequencies for these two repeats: The carrier frequency is 1:90 for FXN (Zamba-Papanicolaou et al. 2009) and 1:178 for the FMR1 premutation (Hantash et al. 2011). Additionally, there was one "control" sample showing an ATXN1 expansion. The final three putative FP samples were identified in the HTT repeat and include a mother and son who were both sized at 30 repeats (bottom of the premutation range), and a third sample with 34 repeats, which is small enough for an individual to be unaffected. Visual inspection of the reads supported the ExpansionHunter calls in these samples.

Although ExpansionHunter is intended for unbiased (e.g., PCR-free) sequence data, 12 of the samples studied here were sequenced with a PCR step in the sample preparation. These comprised nine samples with either a premutation or expansion at the HTT gene and three controls. These samples were correctly classified for the HTT repeat despite the high GC content of this CAG repeat (67\%). Conversely, in these samples the FMR1 repeat length could not be assessed for all but one of these samples: Four samples had no reads covering the repeat and seven were covered very poorly (one to three reads covering the repeat) and produced excessively small repeat lengths. For example, these seven samples were all sized at fewer than 10 repeats, whereas for the other 157 samples sequenced without PCR, only four samples had alleles shorter than 20 repeats and the smallest of these spanned 14 repeats.

\section{Discussion}

We developed a software tool that can identify pathogenic repeat expansions from paired-end, PCR-free WGS data. Comparing against the results obtained with a widely used wet lab protocol for identifying pathogenic repeat expansions in the C9orf72 locus, ExpansionHunter was able to correctly classify all expanded samples as either expansions (208) or potential expansions (4) and 2786 of the 2789 wild-type samples. Some samples are classified as potential expansions, because there is an uncertainty associated with repeats longer than the read length. In a clinical setting, such calls would trigger a follow-up analysis; so all the expansions were flagged in this analysis.

We also demonstrated that our method generalizes to other repeats by correctly identifying the validated
Figure 3. Distribution of ExpansionHunter and lobSTR allele sizes of the C9orf72 repeat in the 1770 samples with $150 \mathrm{bp}$ reads from cohorts one and two, compared with those of the FTLD cohort of 318 samples from a previous study (van der Zee et al. 2013). 


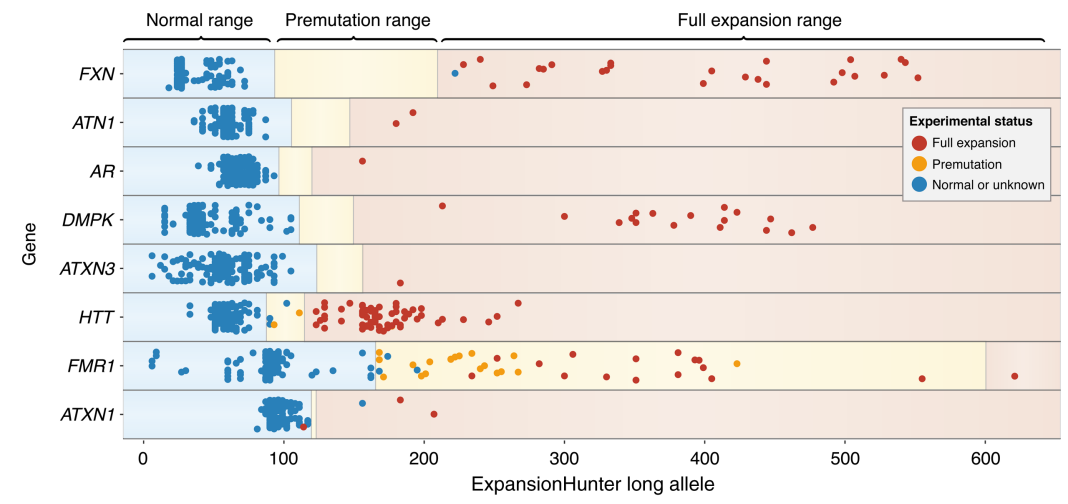

Figure 4. Sizes of the longer repeat alleles predicted by ExpansionHunter in the 152 samples identified as having either a premutation or an expansion at loci associated with eight different diseases and 24 additional control samples. Circles indicate the most-likely repeat length of the longer allele in base pairs for a sample identified with a premutation (orange) or expansion (red), and the blue circles show the predicted repeat lengths for the controls. The controls include samples with measurements showing that they fall in the "normal" range and samples that have a different repeat expansion. Thus, each sample will have one circle for each of the eight repeat expansions. The regions are shaded to indicate the normal ranges (blue), premutation ranges (yellow), and expansion sizes (light red) (McMurray 2010). Additional information is available in Supplemental Tables 7 and 8.

repeats from 152 samples with eight other pathogenic repeat expansions. In total, we examined five repeat motifs (CTG, GAA, CGG, CAG, and GGCCCC) at nine different genomic locations and demonstrated that ExpansionHunter can detect repeat expansions in a variety of sequence contexts. It is particularly important that our method works on the very high (100\%) GC repeats in FMR1 (CGG) and C9orf72 (GGCCCC) genes, where both coverage biases and error rates may be elevated. Comparing our size estimates with Southern blot experiments indicated that our method may underestimate sizes of some very long repeats, particularly those in the FMR1 and $A R$ genes (Fig. 4; Supplemental Table 7). This discrepancy could be due to mosaicism of expanded FMR1 repeats (in fact, several of the samples with FMR1 expansions were identified as mosaic in the Coriell database). In addition to mosaicism, other factors such as higher error rates and GC biases may play a role in causing this method to underestimate the size of these long repeats. Still, the FMR1 expansions were generally sized as being longer compared to the FMR1 premutations, indicating that it may be possible to calibrate the size estimates and account for errors not related to mosaicism. Future work will concentrate on quantifying this behavior and improving the size estimates for these long repeats.

ExpansionHunter is designed for PCR-free WGS data, and comprehensive detection of large pathogenic repeats is unlikely to work with whole-exome sequence data because (1) many repeats of interest are not exonic; and (2) size estimates for large repeats require assumptions about the average number of reads per base. Additionally, some important repeats, like the repeat in FMR1 gene that causes fragile X syndrome, are 100\% GC and are underrepresented in sequence data that includes a PCR step during sample preparation.

If there is no evidence of long repeats with the same repeat motif elsewhere in the genome (e.g., the GGCCCC repeat in C9orf72), both anchored and paired IRRs can be utilized to estimate the full length of the repeat. If, on the other hand, multiple long repeats with the same repeat motif exist, then the size of the repeat is estimated only from anchored in-repeat reads and so is capped by the fragment length. Although the off-target reads im- prove the size estimates produced by this method, most repeats are classified as pathogenic much closer to the read length (e.g., Fig. 4). Because of this, in most cases the anchored reads are sufficient to identify pathogenic repeats and, for example, all 212 of the C9orf72 expanded samples were also identified even when using only the anchored IRRs to estimate the repeat length.

A major benefit of our tool is that it enables researchers to screen for all known repeat expansions using a single whole-genome sequencing run. As the throughput of WGS increases and the cost decreases, WGS may soon become the basis for frontline tests for repeat expansions and other genetic disorders. Theoretically, long reads can also identify many of the longer repeat expansions (Loomis et al. 2013), but those technologies are still too expensive to be routinely used for whole-genome screening. At the same time, because the substitution and indel error rates in these long reads range from $10 \%$ to $30 \%$ (Sović et al. 2016; Bao and Lan 2017), it may be difficult to classify the repeat confidently when its size is close to the normal-premutation or premutation-expansion boundary cutoffs unless the samples are sequenced to high depth.

Because repeat expansions may expand from generation to generation, pathogenic repeats may show little or no linkage disequilibrium with the surrounding variants. Thus, association studies based solely on SNPs may be blind to these highly polymorphic risk alleles. An important result highlighted in Figures 3 and 4 is that ExpansionHunter is able to size both short and long repeats. This will allow researchers to quantify repeat lengths of all STRs genome-wide and discover novel pathogenic repeat expansions agnostically. Although this method can be used to quantify every repeat in the genome, ExpansionHunter is a targeted tool that requires an STR to be specified by its reference coordinates and repeat motif. Additional work is ongoing to develop a genome-wide STR database that ExpansionHunter can use to target any STR genome-wide. As association studies based on high-depth WGS data become more widespread, it will be possible to discover new, previously undetected repeat expansions by genotyping them across the population with ExpansionHunter.

\section{Methods}

\section{Whole-genome sequencing}

Whole-genome shotgun sequencing was performed for all of the samples analyzed in this study. For 1231 of the ALS samples, we used TruSeq DNA PCR-free sample preparation with $100 \mathrm{bp}$ paired-end reads sequenced on Illumina HiSeq 2000 instruments. The remaining 1770 ALS samples, the 128 Coriell samples, and 42 of the Huntington samples used TruSeq DNA PCR-free sample preparation with 150 bp paired-end reads sequenced on Illumina HiSeq X instruments. The final eight samples used TruSeq Nano library preparation with 150 bp paired reads sequenced on Illumina HiSeq X instruments. 


\section{C9orf72 PCR}

Repeat-primed PCR (RP-PCR) was performed on 50-300 ng gDNA with $1 \times$ FastStart Mix (Roche), $0.9 \mathrm{M}$ Betaine, 5\% DMSO, $1 \mathrm{mM}$ $\mathrm{MgCl}_{2}, 0.2 \mathrm{mM}$ 7-deaza-dGTP, $0.6-1.3 \mu \mathrm{M}$ F-primer ([6FAM] AGTCGCTAGAGGCGAAA(GC)), $0.3-1.3 \mu \mathrm{M}$ R-primer (TACG CATCCCAGTTTGAGACGGGGGCCGGGGCCGGGGCC(GGGG)), 0.6-1.3 $\mu \mathrm{M}$ anchor-primer (TACGCATCCCAGTTTGAGACG) in a total volume of $16-30 \mu \mathrm{L}$, with this protocol: $15 \mathrm{~min} 95^{\circ} \mathrm{C} ; 2$ cycles $1 \min 94^{\circ} \mathrm{C}, 1 \min 70^{\circ} \mathrm{C}, 3 \min 72^{\circ} \mathrm{C} ; 3$ cycles $1 \min 94^{\circ} \mathrm{C}, 1 \mathrm{~min}$ $68^{\circ} \mathrm{C}, 3 \min 72^{\circ} \mathrm{C}$; 4 cycles $1 \min 94^{\circ} \mathrm{C}, 1 \min 66^{\circ} \mathrm{C}, 3 \min 72^{\circ} \mathrm{C}$; 5 cycles $1 \min 94^{\circ} \mathrm{C}, 1 \min 64^{\circ} \mathrm{C}, 3 \min 72^{\circ} \mathrm{C} ; 6$ cycles $1 \mathrm{~min}$ $94^{\circ} \mathrm{C}, 1 \min 62^{\circ} \mathrm{C}, 3 \min 72^{\circ} \mathrm{C} ; 7$ cycles $1 \min 94^{\circ} \mathrm{C}, 1 \min 60^{\circ} \mathrm{C}$, $3 \mathrm{~min} 72^{\circ} \mathrm{C} ; 8$ cycles $1 \mathrm{~min} 94^{\circ} \mathrm{C}, 1 \mathrm{~min} 58^{\circ} \mathrm{C}, 3 \mathrm{~min} 72^{\circ} \mathrm{C} ; 5$ cycles $1 \mathrm{~min} 94^{\circ} \mathrm{C}, 1 \mathrm{~min} 56^{\circ} \mathrm{C}, 3 \mathrm{~min} 72^{\circ} \mathrm{C} ; 10 \mathrm{~min} 72^{\circ} \mathrm{C}$. The PCR product was analyzed on an ABI 3730 DNA Analyzer (Applied Biosystems) with PeakScanner software (v1.0). A characteristic stutter pattern was considered evidence of a C9orf72 repeat expansion. Fluorescent PCR was performed as previously described (DeJesus-Hernandez et al. 2011).

\section{Confirmation of C9orf72 RP-PCR results}

The presence of a repeat expansion was determined in a blinded fashion using a two-step PCR protocol (DeJesus-Hernandez et al. 2011). Genomic DNA was PCR amplified with genotyping primers and one fluorescently labeled primer, followed by fragment length analysis with an ABI 3730 DNA analyzer and GeneMapper software (v5). A single PCR fragment could either indicate a homozygous variant or a pathogenic repeat expansion. Subjects with a single PCR fragment were selected for RP-PCR, and PCR products were analyzed with an ABI 3730 DNA Analyzer and GeneMapper software. If the RP-PCR revealed a characteristic stutter pattern, these individuals were screened using Southern blotting techniques, as described previously (DeJesusHernandez et al. 2011). A total of 7-10 $\mu$ g of genomic DNA was digested with XbaI (Promega) and electrophoresed in a 0.8\% agarose gel. DNA was then transferred to a positively charged nylon membrane (Roche), cross-linked, and subsequently hybridized with a digoxigenin (DIG)-labeled probe. Expansions were visualized with anti-DIG antibody (Roche) and CDP-Star substrate (Roche) on X-ray film.

\section{Identifying IRRs}

To test if a read fully consists of the repeat motif, we compared it to the perfect repeat sequence that was the closest match under the shift and reverse complement operations (e.g., a read originating in a CAG repeat can consist of repetitions of either CAG, AGC, GCA in the forward orientation or CTG, TGC, GCT in the reverse orientation). To do the comparison, we defined the weighted purity (WP) score metric that assigns each matching base a score of 1 , each low-quality mismatch a score of 0.5 and each high-quality mismatch a score of -1 . After normalization of the sum of perbase scores for the total read length, the WP ranges from -1 to 1. We defined IRRs as reads that achieve WP of 0.9 or higher (Supplemental Methods).

\section{Identifying off-target regions}

Paired IRRs originating from expanded STRs may align to other genomic locations, especially if the STR is short in the reference genome at the target location. We refer to the loci where IRRs may misalign as off-target regions. Identifying off-target regions enables us to reduce the search for IRRs to a few regions instead of the whole genome. In order to obtain off-target regions for the
C9orf72 repeat, we searched through the 182 samples in cohort one that had an expanded repeat according to the original RPPCR results to identify all the GGGGCC IRRs. The search was performed through the whole genome for read pairs with a low mapping quality $(\mathrm{MAPQ}=0)$ and a weighted purity score of at least 0.9 . The mapping positions of all identified IRRs were merged if they were closer than $500 \mathrm{bp}$, and the resulting 29 loci that were present in five or more samples were designated as off-target regions (Supplemental Fig. 4) and were used to find additional reads from the C9orf72 repeat expansion.

\section{Repeat size estimation from IRRs}

We assume that the probability of observing a read starting at a given base follows the Bernoulli distribution with the probability of success parameter, $\pi$, equal to the ratio of the read depth to the read length. Thus, starting positions of the reads occurring in a given region define a Bernoulli process, and the number of reads starting in the region follows a binomial distribution. If $r$ is the read length, then one of the terminal bases of any IRR must start at least $N-r$ bases away from the flanks of the repeat. The probability of observing $i$ such reads is

$$
P(i, N-r)=\left(\begin{array}{c}
N-r \\
i
\end{array}\right) \pi^{i}(1-\pi)^{N-r-i}
$$

Because we have the estimates for $i$ (the number of IRRs) and $\pi$ (the probability that there is a read starting at a given base), $N$ (the repeat size) can be estimated by $r+i / \pi$. The confidence interval for the repeat size is estimated by the parametric bootstrap method (Rice 2007). The same procedure is used to obtain point estimates and confidence intervals for repeat sizes from flanking reads. The confidence interval is truncated according to the size of the longest repeat sequence observed in a flanking read.

\section{Repeat size determination from spanning reads}

The reads spanning the repeat are identified from all the reads that aligned within $1 \mathrm{~kb}$ of the target repeat region. Each of these reads is tested for the presence of the repeat motif, after which the flanking sequences of the repeat in the read is aligned to the flanking sequences of the repeat in the reference. To be considered spanning, a read must achieve a WP score of 0.9 across the repeat sequence and its flanks. Furthermore, the flanking sequence must have at most two fewer high-quality mismatches or four fewer low-quality mismatches compared to the sequence obtained by extending the repeat. So, if the flanking sequence is similar to the repeat motif, then more flanking sequence is required to identify the end of the repeat and the beginning of the flanking sequence.

\section{Repeat genotyping}

Genotype probabilities for repeats of size up to the read length are calculated using a similar model as the one used for SNPs (Li et al. 2009). Namely, $P(G \mid R)=P(R \mid G) \cdot P(G) / P(R)$ where the genotype $G$ is a tuple of repeat sizes with the number of entries equal to the ploidy of the chromosome containing the repeat. The probability $P(R \mid G)$ is expressed in terms of the probabilities $P\left(r_{i} \mid H_{i}\right)$ for individual reads $r_{i}$ and repeat alleles $H_{i}$ as described ( $\mathrm{Li}$ et al. 2009).

If $r_{i}$ is a spanning read containing $m$ repeat units, $P\left(r_{i} \mid H_{i}=n\right)=$ $\pi \cdot f(m \mid p, n, s)$, where $\pi$ is defined as above ("Repeat size estimation from IRRs"). The frequency function $f$ is defined by $f(m \mid p, n, s) \sim p$ $(1-p)^{d}$, where $m, n, s$ are non-negative integers bounded by the maximum number of repeat units in a read which we denote by $u, p \in(0,1)$ corresponds to the proportion of molecules with 
repeat of the expected size, and $d=|n-m|$ if $|n-m|<s$ and $d=s$ otherwise. Note that $f$ is defined similarly to the geometric frequency function with parameter $d$ representing the deviation from $n$, the expected repeat size (which can be at most $s$ ). If $r_{i}$ is a flanking or in-repeat read containing $m$ repeat units, $P\left(r_{i} \mid H_{i}=n\right)=\pi \cdot \sum_{i=m}^{u} f(i \mid p, n, s)$. In all our analyses, the parameters $p$ and $s$ were set to 0.97 and 5 . The values were chosen to maximize Mendelian consistency of genotype calls in Platinum Genome pedigree samples (Eberle et al. 2017) on an unrelated set of repeats.

We use read-length-sized repeats as a stand-in for repeats longer than the read length. If only one allele is expanded, we estimate the full size of the repeat as described above. If both alleles are expanded, the size intervals are estimated similarly by assuming that between 0 and $50 \%$ of in-repeat reads come from the short allele and between $50 \%$ and $100 \%$ of in-repeat reads come from the long allele.

\section{Software availability}

ExpansionHunter is written in $\mathrm{C}++$, and its source code is included in the Supplemental Materials. The software is licensed under GPLv3.0, and the binaries, source code, and documentation are also freely available at https://github.com/Illumina/Expansion Hunter.

\section{Data access}

WGS for Coriell samples, WGS reads from C9orf72 repeat region for Project MinE samples, and HTT repeat region for Huntington's disease samples from this study have been submitted to the European Genome-phenome Archive (EGA; https:// www.ebi.ac.uk/ega/home) under accession numbers EGAS 00001002462, EGAS00001002598, and EGAS00001002593, respectively. The following cell lines/DNA samples were obtained from the NIGMS Human Genetic Cell Repository at the Coriell Institute for Medical Research: NA04724, NA05446, NA05539, NA05676, NA06477, NA06591, NA05470, NA05438, NA06075, NA04567, NA05164, NA04648, NA05152, NA23378, NA23374, NA23300, NA03986, NA03989, NA03990, NA03696, NA03759, NA04034, NA03697, NA03132, NA03756, NA13716, NA13717, NA03816, NA04079, NA14519, NA15850, NA15847, NA15848, NA16197, NA16200, NA16202, NA16203, NA16205, NA16209, NA16210, NA16212, NA16216, NA16213, NA16215, NA16214, NA16227, NA16229, NA16228, NA16237, NA16243, NA16240, NA16207, NA06895, NA04025, NA04926, NA05131, NA05185, NA09145, NA09237, NA07063, NA07539, NA06890, NA06905, NA07536, NA07540, NA07542, NA06910, NA06894, NA07541, NA07175, NA06889, NA06893, NA06896, NA07538, NA07537, NA06897, NA07174, NA06903, NA07543, NA06852, NA06891, NA06907, NA06906, NA06892, NA06904, NA06968, NA07294, NA09316, NA09317, NA09497, NA07730, CD00014, NA03200, NA20235, NA20238, NA20237, NA20239, NA20242, NA20243, NA20230, NA20232, NA20233, NA20234, NA20236, NA20231, NA20240, NA20241, NA20244, NA07862, NA13509, NA13515, CD00022, NA13507, NA13508, NA13510, NA13511, NA13512, NA13513, NA13514, NA13503, NA13504, NA13505, NA13506, NA06926, NA13536, NA13537, NA06151, NA23709.

\section{Competing interest statement}

Some of the authors are employees of Illumina, Inc.

\section{Acknowledgments}

This work was partially carried out on the Dutch national e-infrastructure with the support of SURF Cooperative. This research was supported by NIH/NINDS P01 NS084974 (M.vB., R.R.), R01 NS080882 (M.vB., R.R.), the Thierry Latran Foundation (M.A.vE., J.H.V., G.H.P.T.), the Netherlands Organization for Health Research and Development (Veni scheme, M.A.vE.), the ALS Foundation Netherlands, the MND Association (UK) (Project MinE, www.projectmine.com), and the W.M. Keck Foundation through the grant "Finding Genetic Modifiers As Avenues to Developing New Therapeutics". Research leading to these results has received funding from the European Community's Health Seventh Framework Programme (FP7/2007-2013) and Horizon 2020 Programme (H2020-PHC-2014-two-stage; grant agreement number 633413). This study was supported by ZonMW under the framework of E-Rare-2, the ERA Net for Research on Rare Diseases (PYRAMID). This is an EU Joint ProgrammeNeurodegenerative Disease Research (JPND) project (STRENGTH, SOPHIA, ALS-CarE). The project is supported through the following funding organizations under the aegis of JPND: UK, Medical Research Council (MR/L501529/1 and ES/L008238/1); Ireland, Health Research Board; Netherlands, ZonMw. A.A.C. receives salary support from the National Institute for Health Research (NIHR) Dementia Biomedical Research Unit at South London and Maudsley NHS Foundation Trust and King's College London. D.E.H. and C.W.N. receive salary from the W.M. Keck Foundation. Samples used in this research were in part obtained from the UK National DNA Bank for MND Research, funded by the MND Association and the Wellcome Trust. We acknowledge sample management undertaken by Biobanking Solutions funded by the Medical Research Council at the Centre for Integrated Genomic Medical Research, University of Manchester.

\section{References}

Akimoto C, Volk AE, van Blitterswijk M, Van den Broeck M, Leblond CS, Lumbroso S, Camu W, Neitzel B, Onodera O, van Rheenen W, et al. 2014. A blinded international study on the reliability of genetic testing for GGGGCC-repeat expansions in C9orf72 reveals marked differences in results among 14 laboratories. J Med Genet 51: 419-424.

Ashley EA. 2015. The precision medicine initiative: a new national effort. JAMA 313: 2119-2120.

Ashley EA. 2016. Towards precision medicine. Nat Rev Genet 17: 507-522.

Bao E, Lan L. 2017. HALC: high throughput algorithm for long read error correction. BMC Bioinformatics 18: 204.

Benjamini Y, Speed TP. 2012. Summarizing and correcting the GC content bias in high-throughput sequencing. Nucleic Acids Res 40: e72.

Buchman VL, Cooper-Knock J, Connor-Robson N, Higginbottom A, Kirby J, Razinskaya OD, Ninkina N, Shaw PJ. 2013. Simultaneous and independent detection of C9ORF72 alleles with low and high number of GGGGCC repeats using an optimised protocol of Southern blot hybridisation. Mol Neurodegener 8: 12.

Chen X, Schulz-Trieglaff O, Shaw R, Barnes B, Schlesinger F, Källberg M, Cox AJ, Kruglyak S, Saunders CT. 2016. Manta: rapid detection of structural variants and indels for germline and cancer sequencing applications. Bioinformatics 32: 1220-1222.

Church DM, Schneider VA, Steinberg KM, Schatz MC, Quinlan AR, Chin CS, Kitts PA, Aken B, Marth GT, Hoffman MM, et al. 2015. Extending reference assembly models. Genome Biol 16: 13 .

DeJesus-Hernandez $\mathrm{M}$, Mackenzie IR, Boeve BF, Boxer AL, Baker $\mathrm{M}$, Rutherford NJ, Nicholson AM, Finch NA, Flynn H, Adamson J, et al. 2011. Expanded GGGGCC hexanucleotide repeat in noncoding region of C9ORF72 causes chromosome 9p-linked FTD and ALS. Neuron 72: 245-256.

DePristo MA, Banks E, Poplin R, Garimella KV, Maguire JR, Hartl C, Philippakis AA, del Angel G, Rivas MA, Hanna M, et al. 2011. A framework for variation discovery and genotyping using next-generation DNA sequencing data. Nat Genet 43: 491-498.

Dürr A, Cossee M, Agid Y, Campuzano V, Mignard C, Penet C, Mandel JL, Brice A, Koenig M. 1996. Clinical and genetic abnormalities in patients with Friedreich's ataxia. N Engl J Med 335: 1169-1175.

\section{Genome Research}

www.genome.org 
Eberle MA, Fritzilas E, Krusche P, Källberg M, Moore BL, Bekritsky MA, Iqbal Z, Chuang HY, Humphray SJ, Halpern AL, et al. 2017. A reference data set of 5.4 million phased human variants validated by genetic inheritance from sequencing a three-generation 17-member pedigree. Genome Res 27: 157-164.

Gatchel JR, Zoghbi HY. 2005. Diseases of unstable repeat expansion: mechanisms and common principles. Nat Rev Genet 6: 743-755.

Gijselinck I, Van Langenhove T, van der Zee J, Sleegers K, Philtjens S, Kleinberger G, Janssens J, Bettens K, Van Cauwenberghe C, Pereson S, et al. 2012. A C9orf72 promoter repeat expansion in a FlandersBelgian cohort with disorders of the frontotemporal lobar degeneration-amyotrophic lateral sclerosis spectrum: a gene identification study. Lancet Neurol 11: 54-65.

Gijselinck I, Van Mossevelde S, van der Zee J, Sieben A, Engelborghs S, De Bleecker J, Ivanoiu A, Deryck O, Edbauer D, Zhang M, et al. 2016. The C9orf72 repeat size correlates with onset age of disease, DNA methylation and transcriptional downregulation of the promoter. Mol Psychiatry 21: 1112-1124.

Gymrek M, Golan D, Rosset S, Erlich Y. 2012. lobSTR: a short tandem repeat profiler for personal genomes. Genome Res 22: 1154-1162.

Hantash FM, Goos DM, Crossley B, Anderson B, Zhang K, Sun W, Strom CM. 2011. FMR1 premutation carrier frequency in patients undergoing routine population-based carrier screening: insights into the prevalence of fragile X syndrome, fragile $\mathrm{X}$-associated tremor/ataxia syndrome, and fragile $\mathrm{X}$-associated primary ovarian insufficiency in the United States. Genet Med 13: 39-45.

Iqbal Z, Caccamo M, Turner I, Flicek P, McVean G. 2012. De novo assembly and genotyping of variants using colored de Bruijn graphs. Nat Genet 44: $226-232$.

Kronquist KE, Sherman SL, Spector EB. 2008. Clinical significance of tri-nucleotide repeats in Fragile X testing: a clarification of American College of Medical Genetics guidelines. Genet Med 10: 845-847.

Li H. 2015. FermiKit: assembly-based variant calling for Illumina resequencing data. Bioinformatics 31: 3694-3696.

Li H, Durbin R. 2009. Fast and accurate short read alignment with BurrowsWheeler transform. Bioinformatics 25: 1754-1760.

Li R, Li Y, Fang X, Yang H, Wang J, Kristiansen K, Wang J. 2009. SNP detection for massively parallel whole-genome resequencing. Genome Res 19: 1124-1132.

Loomis EW, Eid JS, Peluso P, Yin J, Hickey L, Rank D, McCalmon S, Hagerman RJ, Tassone F, Hagerman PJ. 2013. Sequencing the unse- quenceable: expanded CGG-repeat alleles of the fragile $\mathrm{X}$ gene. Genome Res 23: 121-128.

Marx V. 2015. The DNA of a nation. Nature 524: 503-505.

McMurray CT. 2010. Mechanisms of trinucleotide repeat instability during human development. Nat Rev Genet 11: 786-799.

Meienberg J, Bruggmann R, Oexle K, Matyas G. 2016. Clinical sequencing: Is WGS the better WES? Hum Genet 135: 359-362.

Narzisi G, Schatz MC. 2015. The challenge of small-scale repeats for indel discovery. Front Bioeng Biotechnol 3: 8.

Raczy C, Petrovski R, Saunders CT, Chorny I, Kruglyak S, Margulies EH, Chuang HY, Källberg M, Kumar SA, Liao A, et al. 2013. Isaac: ultra-fast whole-genome secondary analysis on Illumina sequencing platforms. Bioinformatics 29: 2041-2043.

Renton AE, Majounie E, Waite A, Simón-Sánchez J, Rollinson S, Gibbs JR, Schymick JC, Laaksovirta H, van Swieten JC, Myllykangas L, et al. 2011. A hexanucleotide repeat expansion in C9ORF72 is the cause of chromosome 9p21-linked ALS-FTD. Neuron 72: 257-268.

Rice JA. 2007. Mathematical statistics and data analysis. Cengage Learning Inc., Boston, MA.

Sović I, Šikić M, Wilm A, Fenlon SN, Chen S, Nagarajan N. 2016. Fast and sensitive mapping of nanopore sequencing reads with GraphMap. Nat Commun 7: 11307.

van der Zee J, Gijselinck I, Dillen L, Van Langenhove T, Theuns J, Engelborghs S, Philtjens S, Vandenbulcke M, Sleegers K, Sieben A, et al. 2013. A pan-European study of the C9orf72 repeat associated with FTLD: geographic prevalence, genomic instability, and intermediate repeats. Hum Mutat 34: 363-373.

Weisenfeld NI, Yin S, Sharpe T, Lau B, Hegarty R, Holmes L, Sogoloff B, Tabbaa D, Williams L, Russ C, et al. 2014. Comprehensive variation discovery in single human genomes. Nat Genet 46: 1350-1355.

Wexler NS, Lorimer J, Porter J, Gomez F, Moskowitz C, Shackell E, Marder K, Penchaszadeh G, Roberts SA, Gayán J, et al. 2004. Venezuelan kindreds reveal that genetic and environmental factors modulate Huntington's disease age of onset. Proc Natl Acad Sci 101: 3498-3503.

Zamba-Papanicolaou E, Koutsou P, Daiou C, Gaglia E, Georghiou A, Christodoulou K. 2009. High frequency of Friedreich's ataxia carriers in the Paphos district of Cyprus. Acta Myol 28: 24-26.

Received June 1, 2017; accepted in revised form August 28, 2017. 


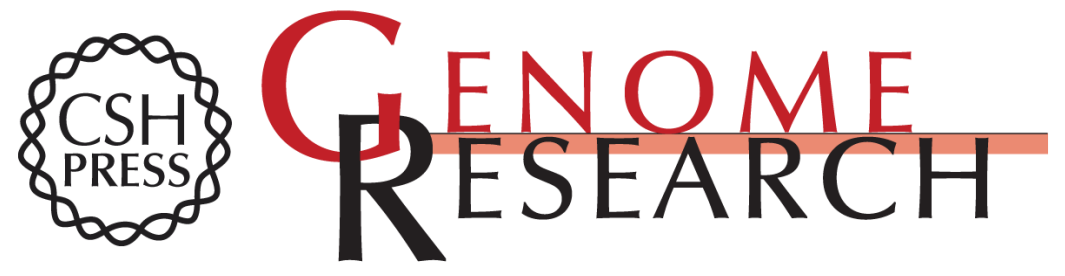

\section{Detection of long repeat expansions from PCR-free whole-genome sequence data}

Egor Dolzhenko, Joke J.F.A. van Vugt, Richard J. Shaw, et al.

Genome Res. 2017 27: 1895-1903 originally published online September 8, 2017

Access the most recent version at doi:10.1101/gr.225672.117

Supplemental Material

References

Open Access

Creative Commons

License

Email Alerting Service
http://genome.cshlp.org/content/suppl/2017/10/13/gr.225672.117.DC1

This article cites 34 articles, 6 of which can be accessed free at: http://genome.cshlp.org/content/27/11/1895.full.html\#ref-list-1

Freely available online through the Genome Research Open Access option.

This article, published in Genome Research, is available under a Creative Commons License (Attribution 4.0 International), as described at http://creativecommons.org/licenses/by/4.0/.

Receive free email alerts when new articles cite this article - sign up in the box at the top right corner of the article or click here.

\section{Affordable, Accurate Sequencing.}

